\title{
A COMPARATIVE STUDY OF STOCHASTIC QUADRATIC PROGRAMMING AND OPTIMAL CONTROL MODEL IN PRODUCTION-INVENTORY SYSTEM WITH STOCHASTIC DEMAND
}

\author{
Ali Khaleel Dhaiban \\ Received October 11, 2016 / Accepted March 24, 2017
}

\begin{abstract}
This study compares the optimal control model and stochastic quadratic programming (SQP) model of a production-inventory system. A single product, without shortage in the case of a periodic-review policy with stochastic demand and deterioration rate as a function of time, is discussed. The items are subjected to deterioration via storage and the inventory goal level as a function of production. Demand is represented by a stochastic differential equation, converted to a stochastic constraint, and then to a deterministic constraint. We derive the optimality conditions of optimal control model and formulate three models of SQP. The effect of stochastic demand on the production rate and inventory level is then illustrated. Our numerical results appear to suggest that control of inventory level is better in the case of SQP. Furthermore, the total cost is similar in the three models of SQP - despite a difference in production rates and inventory levels.
\end{abstract}

Keywords: optimal control, production-inventory system, stochastic quadratic programming.

\section{INTRODUCTION}

Production planning needs to take many factors into account, in order to realize maximum profitability. Inventory, which is one of these factors, has emerged in the last decade. The balance between production process and inventory level, to hedge demand with the lowest cost, is very important in any production-inventory system. Many mathematical models, such as optimal control and mathematical programming, have been developed to deal with this system. Productioninventory systems face many problems, such as the life age of products during storage and the determination of exogenous demand. In practice, demand is often not specified, as it relies on customers and competition. Therefore, a production-inventory system with stochastic demand was developed.

Numerous researchers have investigated inventory systems with stochastic demand. Kleywegt et al. (2004), Mubiru (2014) and Feng et al. (2015) have formulated the Markov decision process 
to describe stochastic demand. Kleywegt et al. (2004) discussed a routing problem, and proposed a method to solve that problem. The target was to minimize the total cost by determining the replenishment amount, and selecting a suitable method of transportation. Mubiru (2014) detailed the order policy on milk powder in supermarkets to minimize the total cost of ordering and holding stock, in addition to the shortage cost. The optimal policy of replenishment, and its structure in inventory systems with multiple products, neglect lead time, and discount rate, was investigated by Feng et al. (2015). He proposed a policy that clarified the stochastic demand effect on the optimal policy structure. Levi et al. (2007) considered two problems of stochastic inventory; periodic-review and lot-sizing, with single item and location. The goal was to balance between the costs of excess inventory and backlog demand, in order to minimize total costs. Hurley et al. (2007) extended the Levy model by proposing two policies to determine the lower and upper bounds of inventory depending on the techniques of cost-balancing and myopic-like. Wanke (2010) investigated two rules of demand allocation with lead time and demand as random variables that follow a normal distribution. He compared two rules based on safety stock, total stock and total cost. Two optimization models with defective items and normal distribution of demand; fuzzy and intuitionistic fuzzy were investigated by Banerjee (2011). The author discussed the chance-constraints model with multi objectives and demand that adhered to uniform and exponential distributions. Also, using two cases of defective items, Bhowmick \& Samanta (2012) addressed the inventory model with a single period, partial backlogging, and stochastic demand, depending on the price to determine the order quantity. Kim \& Jeong (2012) developed a mathematical model of buyer-supplier with a single item, demand normally distributed, and periodic-review to find the optimal length of cycle. Demand, as a simple Poisson process in the continuous- review of the multistage inventory system, was discussed by Hu \& Yang (2014). They modified a policy of echelon $(r, Q)$ replenishment, for any stage, based on previous stages, and clarified the results of a multi-stage system based on a two-stage system's results. The works of Kumar et al. (2011), Castellano (2015), Mubiru (2015) and Soysal (2016), covered many aspects of this topic.

An optimal control model of the production-inventory system with stochastic demand has been investigated by many researchers. Ouaret et al. (2011) and Yi et al. (2013) have investigated optimal control model with demand as a stochastic differential equation, backlog demand and finite capacity. The goal was to minimize the total cost of the quadratic objective function that represented penalties of the deviate inventory level and the production rate from its goals. Ouaret et al. (2011) used the Pontryagin's maximum principles, while Yi et al. (2013) used the HamiltonJacobi-Bellman equation to determine the explicit solution. Germs et al. (2013) formulated an optimal stopping problem to the production planning of a single product and a single machine, using demand as a compound Poisson process. They simplified their problem to a free boundary problems, and showed that their approach could be applied to other models, such as lost-sales. Kutzner \& Kiesmuller (2013) developed a model to minimize the total cost of inventory, includes inspection and backorder costs, in the case of periodic review. A deterministic model of mean value problem (MVP), which is equivalent to the optimal control model with normal distribution of demand, and chance constraints of inventory and production, was developed by Silva Filho (2014). 
Another mathematical model, which was used to plan a production-inventory system, is stochastic mathematical programming. Tarim et al. (2006) developed stochastic linear programming with a discrete distribution of demand and scenario trees for stochastic constraints to minimize the expected total cost of shortage and scrap in an inventory system. Also with stochastic linear programming, Zhang et al. (2006) applied the inventory system to fashion production with a single period, multiple retailers and stocking echelons; where the first echelon was for raw materials and finished goods were the last echelon. Rossi et al. (2007) studied the replenishment cycle in a production-inventory system with multiple periods, normal distribution of demand, and shortage. The paper took into account the four fixed costs of holding, procurement, ordering, and shortage. A stochastic demand modelled as a scenario tree, such as the one in Tarim et al. (2006), with uncertainty in the equality of the raw materials, multiple periods, and multiple products in sawmill production, was addressed by Kazemi Zanjani et al. (2010). Dogru et al. (2010) and Reiman \& Wang (2015) have considered an assemble system with multiple components that was used to assemble multiple products with demand as a compound Poisson process and fixed lead time of component replenishment. They developed two-stage stochastic programming to minimize inventory total cost. The effect of risk-pooling on the inventory management of chemical complexes was clarified by You \& Grossmann (2011). They formulated an inventory model as mixed-integer nonlinear to determine the optimal rates of feedstocks purchase, production, and inventory, as well as the sale of chemicals. Furthermore, with a mixed-integer but linear model, Chotayakul \& Punyangarm (2016) addressed the lot-sizing model using cost, capacity, and time of production: in addition to time and cost of setup, based on the machines used.

Our model contributes knowledge to the literature in several ways. The main contribution clarifies the difference of inventory control according to the two models i.e., optimal control and SQP. This is followed by dealing with deteriorating items in the inventory system, by formulating an SQP model. This is in addition to inventory goal level, as a function of production rather than being a constant, which has been addressed by most previous studies. The last step addresses optimal control using stochastic demand, by developing an optimal control model with equivalent deterministic demand, rather than a stochastic optimal control.

This paper is organized in the following order. Section 2.1 will introduce the notations and assumptions involved in the optimal inventory model. Section 2.2 will illustrate the stochastic demand. Sections 2.3 and 2.4 will discuss the formulation of the optimal control model and the derivation of the optimality conditions of the periodic-review system. Section 2.5 will detail the SQP model. Section 3 will illustrate the results of the two aforementioned models. The final section will summarize our findings and suggest future researches.

\section{MATERIALS AND METHODS}

\subsection{Notations and Assumptions Involved In the Model}

\subsubsection{Notations}

The following variables and parameters are used: 
$T=$ The length of the planning horizon $(T>0)$.

$Y(t)=$ The inventory level at time $t$.

$N(t)=$ The production rate at time $t$.

$D(t)=$ The stochastic demand rate for the production at time $t$.

$D(0)=$ The initial demand.

$\delta(t)=$ The deterioration rate, which depends on the time.

$\hat{y}(t)=$ The inventory goal level, which depends on the production.

$\hat{n}(t)=$ The production goal rate.

$Y(0)=$ The initial inventory level.

$h=$ A penalty is incurred when the inventory level deviates from its goal level $(h>0)$.

$k=$ A penalty is incurred when the total production rate to deviate from its goal rate $(k>0)$.

\subsubsection{Assumptions}

We took into account the following:

1. A firm can produce a certain product, sell some, and stack the rest in a warehouse.

2. The stochastic demand rate.

3. The firm has set an inventory goal level and a production goal rate.

4. Production rates are positive to satisfy demand and achieve a specific level of inventory.

5. No shortage and items are subjected to deterioration through storage.

6. Neglect lead time.

\subsection{The Stochastic Demand}

Previous studies that dealt with inventory system have taken into account the many types of demand; for example, stochastic demand (Mubiru, 2014; Feng et al., 2015), demand as a compound Poisson process (Dogru et al., 2010; Reiman \& Wang, 2015) and demand depend on the return products (Raupp et al., 2015). In this study, the demand is represented by the following stochastic differential equation (SDE) (Kiesmüller, 2003; Ouaret et al., 2011; Yi et al., 2013):

$$
d D(t)=\mu d t+\sigma d \beta(t)
$$

where

$\mu=$ The drift coefficient (constant).

$\sigma \quad=$ The diffusion coefficient (constant).

$\beta(t)=$ The Brownian motion. 
The solution of the Eq. (1) is as follows (Wiersema, 2008):

$$
\begin{aligned}
& \int_{0}^{t} d D(s)=\int_{0}^{t} \mu d s+\int_{0}^{t} \sigma d \beta(s) \\
& D(t)-D(0)=\mu(t-0)+\sigma\{\beta(t)-\beta(0)\} \\
& D(t)=D(0)+\mu t+\sigma \beta(t)
\end{aligned}
$$

Eq. (2) includes two parts; the first part is deterministic $D(0)+\mu t$ and the second part is a random variable $\beta(t)$ that follows a normal distribution with mean zero and variance $t$.

According to Karki et al. (2014), the stochastic constraint (2) can be converted to a deterministic constraint using the median of $\beta(t)$. In normal distribution the median is equal to the mean, which means that the median is equal to zero.

$$
D(t)=D(0)+\mu t
$$

Eq. (3) is used in the optimal control model and the quadratic programming model that is equivalent to the SQP model.

Another case to the SQP is rewriting Eq. (2) as a chance constraint by considering the Brownian motion as a $\mathrm{b}$ vector in the mathematical programming:

$$
\begin{gathered}
D(t)=D(0)+\mu t+\sigma \beta(t) \\
\frac{D(t)-D(0)-\mu t}{\sigma}=\beta(t) \\
\operatorname{Pr}_{r}\left\{\frac{D(t)-D(0)-\mu t}{\sigma} \geq \beta(t)\right\} \geq p_{t}
\end{gathered}
$$

or

$$
P_{r}\left\{\frac{D(t)-D(0)-\mu t}{\sigma} \leq \beta(t)\right\} \geq p_{t}
$$

where $p$ is the success probability.

If $p=0.99$, it means that the constraint will be achieved with only one percentage of failure (Prékopa, 2013). According to Shapiro \& Dentcheva (2014), the deterministic constraint that is equivalent to the stochastic constraint (4) is as follows:

$$
\begin{aligned}
& P_{r}\left\{\frac{D(t)-D(0)-\mu t-0}{\sigma \sqrt{t}} \leq \frac{\beta(t)-0}{\sqrt{t}}\right\} \geq p_{t} \\
& \varnothing\left\{\frac{D(t)-D(0)-\mu t}{\sigma \sqrt{t}}\right\} \geq p_{t}
\end{aligned}
$$

where $\varnothing(\cdot)$ is the cumulative distribution function (CDF) of standard normal distribution. 
Eq. (6) can be written as follows:

$$
D(t) \geq D(0)+\mu t+\sigma \sqrt{t} \varnothing^{-1}\left(p_{t}\right)
$$

where $\varnothing^{-1}(\cdot)$ is the inverse of CDF of standard normal distribution, and it is taken directly from the standard normal distribution table.

In the same manner, the deterministic constraint that is equivalent to the stochastic constraint (5) is as follows:

$$
\begin{array}{r}
1-\varnothing\left\{\frac{D(t)-D(0)-\mu t}{\sigma \sqrt{t}}\right\} \geq p_{t} \\
D(t) \leq D(0)+\mu t+\sigma \sqrt{t} \varnothing^{-1}\left(1-p_{t}\right)
\end{array}
$$

\subsection{Optimal control model}

The objective function can be expressed as the quadratic form to minimize (Sethi \& Thompson, 2000):

$$
2 J=\sum_{t=0}^{T-1} h\{Y(t)-\hat{y}(t)\}^{2}+k\{N(t)-\hat{n}(t)\}^{2}
$$

subject to the state equation

$$
\Delta Y(t)=N(t)-D(t)-\delta(t) Y(t) ; \quad t=0, \ldots, T-1
$$

and positive constraint

$$
N(t)>0 ; \quad t=0,1, \ldots, T-1
$$

with initial and terminal conditions

$$
\begin{aligned}
& Y(0)=y_{0} \\
& \lambda(T)=0
\end{aligned}
$$

where $\Delta Y(t)=Y(t+1)-Y(t)$ is called the difference operator.

\subsection{Optimality Conditions and Solution of the Model}

Many pervious works have dealt with the inventory goal level as a constant, and in this paper, it is a function of production:

$$
\begin{gathered}
\hat{y}(t+1)=\varphi \hat{n}(t) ; \quad t=0, \ldots, T-1 ; \quad 0<\varphi<1 \\
\hat{y}(0)=\hat{y}_{0}
\end{gathered}
$$

Eq. (12) depends on the state variable $Y(t)$, and its measured at the beginning period $t$, while during the period, the control variable $N(t)$ is determined.

From the state equation (10), the goals of production and inventory are given by:

$$
\Delta \hat{y}(t)=\hat{n}(t)-D(t)-\delta(t) \hat{y}(t) ; \quad t=0, \ldots, T-1
$$


Substituting Eq. (12) into Eq. (13) yields:

$$
\hat{n}(t)=\frac{D(t)}{1-\varphi}-\frac{1-\delta(t)}{1-\varphi} \hat{y}(t) ; \quad t=0, \ldots, T-1
$$

The Lagrangian function is:

$$
\begin{aligned}
& L=\sum_{t=0}^{T-1}-\frac{1}{2}\left[h\{Y(t)-\hat{y}(t)\}^{2}+k\{N(t)-\hat{n}(t)\}^{2}\right]+\sum_{t=0}^{T-1} \lambda(t+1) \\
& \times[N(t)-D(t)-\delta(t) Y(t)-Y(t+1)+Y(t)]+\sum_{t=0}^{T-1} Z(t) N(t) .
\end{aligned}
$$

A Hamiltonian function defined as:

$$
\begin{gathered}
H(t)=\frac{-1}{2}\left[h\{Y(t)-\hat{y}(t)\}^{2}+k\{N(t)-\hat{n}(t)\}^{2}\right] \\
+\lambda(t+1)[N(t)-D(t)-\delta(t) Y(t)] ; t=0, \ldots, T-1
\end{gathered}
$$

We can write Eq. (15) as follow:

$$
L=\sum_{t=0}^{T-1}\{H(t)-\lambda(t+1)(Y(t+1)-Y(t))\}+\sum_{t=0}^{T-1} Z(t) N(t)
$$

Lagrange multiplier $Z(t)$ satisfy the complementary slackness conditions:

$$
Z(t) \geq 0 ; \quad Z(t) N(t)=0 .
$$

From Eqs. (11) and (18), we get:

$$
Z(t)=0
$$

Equations $(11 \& 16)$ are concave in $N(t)$, so Eqs. (16 \& 18) are the necessary and sufficient conditions for maximizing the Hamiltonian problem.

Now, differentiate Eq. (17) with respect to $Y(t)$ yields:

$$
\Delta \lambda(t)=h\{Y(t)-\hat{y}(t)\}+\lambda(t+1) \delta(t) ; t=0, \ldots, T-1
$$

Differentiate Eq. (17) with respect to $N(t)$, yields:

$$
N(t)=\hat{n}(t)+\frac{1}{k} \lambda(t+1) ; t=0,1, \ldots, T-1
$$

Substituting Eqs. (13 \& 21) into Eq. (10), yields:

$$
\Delta Y(t)=\hat{y}(t+1)-\hat{y}(t)-\delta(t)\{Y(t)-\hat{y}(t)\}+\frac{1}{k} \lambda(t+1) ; t=0, \ldots, T-1
$$


From Eqs. (20 \& 22), we obtained on the following system of difference equations

$$
\left.\begin{array}{l}
\Delta Y(t)=-\delta(t)\{Y(t)-\hat{y}(t)\}+\frac{1}{k} \lambda(t+1) ; t=0, \ldots, T-1 \\
\Delta \lambda(t)=h\{Y(t)-\hat{y}(t)\}+\lambda(t+1) \delta(t) ; t=0, \ldots, T-1
\end{array}\right\}
$$

This equations system (23) can be solved numerically by using Excel with initial condition $Y(0)=y_{0}$ and the terminal condition $\lambda(T)=0$, and then find the production rate from Eq. (21).

\subsection{Stochastic Quadratic Programming (SQP) Formulation}

In this section, we formulate the SQP problem with quadratic cost function subjected to equality and inequality linear and stochastic constraints (Dostál, 2009).

The objective function is represented by the quadratic form to minimize penalties are incurred when the inventory level and production rates deviate from its goals. The objective function is similar to the objective function of the optimal control, which means Eq. (9). The constraints are as follow:

1. The inventory level constraints are:

$$
Y(t+1)-N(t)+D(t)-\{1-\delta(t)\} Y(t)=0 ; t=0, \ldots, T-1
$$

Eq. (24) represents the inventory levels, which is equal to the production, subtract demand and deterioration.

2. The stochastic demand constraint represents three cases, which means three models of SQP. The first model with Eq. (2), the second model with Eq. (4), and the last model with Eq. (5).

3. Assuming the initial demand constraint is:

$$
D(0)=100
$$

4. Inventory goal level constraints are:

$$
\begin{aligned}
& \hat{y}(t+1)-\varphi \hat{n}(t)=0 ; \quad t=0, \ldots, T-1 \\
& \hat{y}(0)-\hat{y}_{0}=0 \\
& \left.\begin{array}{ll}
Y(t)-\hat{y} \geq 0 ; t=0, \ldots, T-1 ; & \hat{y}>Y(0) \\
\hat{y}-Y(t) \geq 0 ; t=0, \ldots, T-1 ; & \hat{y}<Y(0)
\end{array}\right\}
\end{aligned}
$$

Eq. (26) represents inventory goal level, which is equal to the function of production. Meanwhile, Eq. (27) leads to minimize the deviation of inventory level from its goals. 
5. The initial inventory level constraint is:

$$
Y(0)=y_{0}
$$

6. Production goal rate constraints that are similar to Eq. (14):

$$
\hat{n}(t)-\frac{D(t)}{1-\varphi}+\frac{1-d(t)}{1-\varphi} \hat{y}(t)=0 ; \quad t=0, \ldots, T-1
$$

7. Production rate constraints are:

$$
\left.\begin{array}{l}
N(t)-\hat{n}(t) \geq 0 ; t=0,1, \ldots, T-1 \\
N(t)>0 ; t=0,1, \ldots, T-1
\end{array}\right\}
$$

Eq. (30) leads to minimize the deviation of production rate from its goals.

8. Deterioration constraint, which is equal to the function of time, is:

$$
\delta(t)-0.03 t=0 ; t=0, \ldots, T-1
$$

To find a solution of the SQP models, we must replace the stochastic demand constraints by its equivalent deterministic constraints. Therefore, Eq. (2) replaced by Eq. (3), Eq. (4) replaced by Eq. (7), and Eq. (5) replaced by Eq. (8).

By assuming $p_{t}=0.99$, it yields:

$$
\begin{aligned}
& \varnothing^{-1}(0.99)=2.323 \\
& \varnothing^{-1}(0.01)=-2.323
\end{aligned}
$$

From Eqs. (7 \& 8), we get:

$$
\begin{aligned}
& D(t) \geq D(0)+\mu t+\sigma \sqrt{t} * 2.323 ; \quad t=1, \ldots, T-1 \\
& D(t) \leq D(0)+\mu t+\sigma \sqrt{t} *-2.323 ; \quad t=1, \ldots, T-1
\end{aligned}
$$

\section{RESULTS AND DISCUSSION}

Consider an inventory system with the following parameter values $y_{0}=10$ item; $\hat{y}(0)=5$; $T=5$ weaks; $k=25 \$ ; h=10 \$ ; \varphi=0.2 ; \delta(t)=0.03 t ; \mu=25 ; \sigma=10$.

\subsection{Solution of the optimal control model}

By using the goal seek function in Excel, we find the solution of the system (23):

The simulation results given in Figures $(1 \& 2)$ show that the inventory level and the production rate are converging to its goal level over time, as desired. Our results consist with results that 


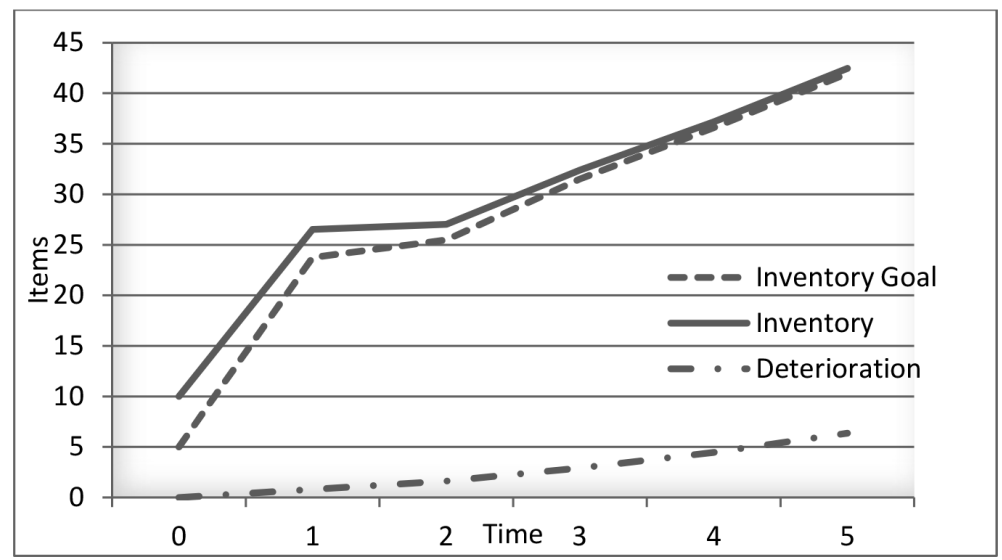

Figure 1 - The inventory level, according to the optimal control model.

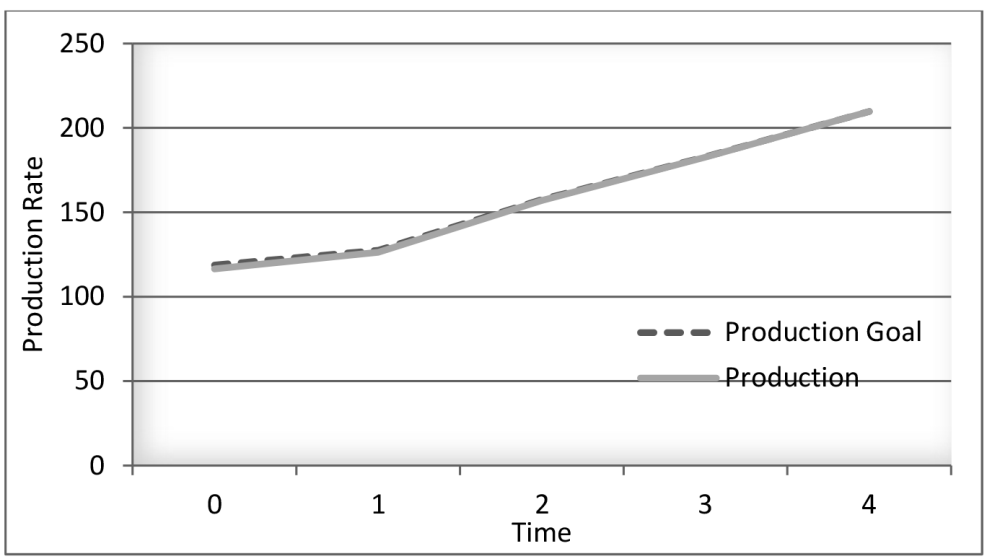

Figure 2 - The production rate, according to the optimal control model.

Table 1 - Solution of the optimal control model.

\begin{tabular}{|c|c|c|c|c|c|c|}
\hline \multirow{2}{*}{ M.V. } & \multicolumn{7}{|c|}{ Time } \\
\cline { 2 - 7 } & 0 & 1 & 2 & 3 & 4 & 5 \\
\hline$Y$ & 10.00 & 26.53 & 27.03 & 32.40 & 37.16 & 42.46 \\
\hline$\hat{y}$ & 5.00 & 23.75 & 25.49 & 31.51 & 36.58 & 41.95 \\
\hline$\hat{n}$ & 118.75 & 127.45 & 157.55 & 182.91 & 209.76 & \\
\hline$N$ & 116.53 & 126.30 & 156.98 & 182.68 & 209.76 & \\
\hline$\delta Y$ & 0.00 & 0.80 & 1.62 & 2.92 & 4.46 & 6.37 \\
\hline$\lambda$ & -105.61 & -55.61 & -28.71 & -14.11 & -5.76 & 0.00 \\
\hline$J$ & \multicolumn{7}{|c|}{$264 \$$} \\
\hline
\end{tabular}


found by Sethi \& Thompson (2000) and Pan \& Li (2014), which means inventory level and production rates as close as to their goals over time, regardless a difference in the assumptions of the models.

The following can be deduced from Table 1:

1. Depending on the value of penalty costs, the convergence between the production rate and its goal is more than the convergence between the inventory level and its goal.

2. The production rate is equal to its goal in the fourth week, due to the terminal condition of $\lambda(5)=0$.

3. The total cost is $264 \$$.

\subsection{Solution of the SQP model}

To find the solution of the SQP problem, we use MatLab (version 8.5).

Table 2 - Solution of the SQP model.

\begin{tabular}{|c|c|c|c|c|c|c|}
\hline \multirow{2}{*}{ M.V. } & \multicolumn{7}{|c|}{ Time } \\
\cline { 2 - 7 } & 0 & 1 & 2 & 3 & 4 & 5 \\
\hline$Y$ & 10.00 & 23.75 & 25.49 & 31.51 & 36.58 & 41.95 \\
\hline$\hat{y}$ & 5.00 & 23.75 & 25.49 & 31.51 & 36.58 & 41.95 \\
\hline$\hat{n}$ & 118.75 & 127.45 & 157.55 & 182.91 & 209.76 & \\
\hline$N$ & 118.75 & 127.45 & 157.55 & 182.91 & 209.76 & \\
\hline$\delta Y$ & 0.00 & 0.71 & 1.53 & 2.84 & 4.39 & 6.29 \\
\hline$J$ & \multicolumn{7}{|c|}{$125 \$$} \\
\hline
\end{tabular}

The following can be deduced from Table 2:

1. The inventory level and the production rate are both equal to its goal.

2. The total cost is $125 \$$, which means that the quadratic programming model is better than the optimal control model.

3. The deterioration rate in the quadratic programming is less than in the optimal control model due to its reliance on the inventory levels.

4. The production rate is similar in two models at the end of the planning period.

\subsection{Inventory level according to the two models}

In this subsection, we compare between the inventory level, according to the optimal control (OC) and quadratic programming (QP). The target of the objective function is to minimize the deviation of inventory level from its goal. 


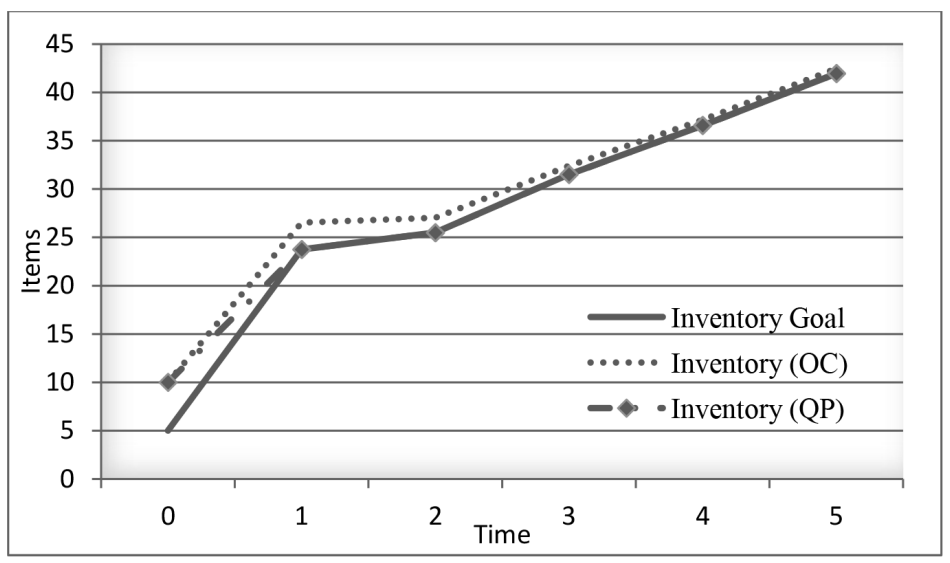

Figure 3 - The inventory level, according to the optimal control and quadratic programming.

In the quadratic programming model, the convergence between inventory level and its goal is better than an optimal control model. This means the total cost of quadratic programming is less than the total cost of optimal control.

\subsection{The effect of the stochastic demand on the results}

This section presents the effect of the three cases of stochastic demand on the production rate and inventory level.

Figure 4 shows the demand rate becoming higher when the case of stochastic constraint is greater than or equal, (see Eq. 7), and vice versa.

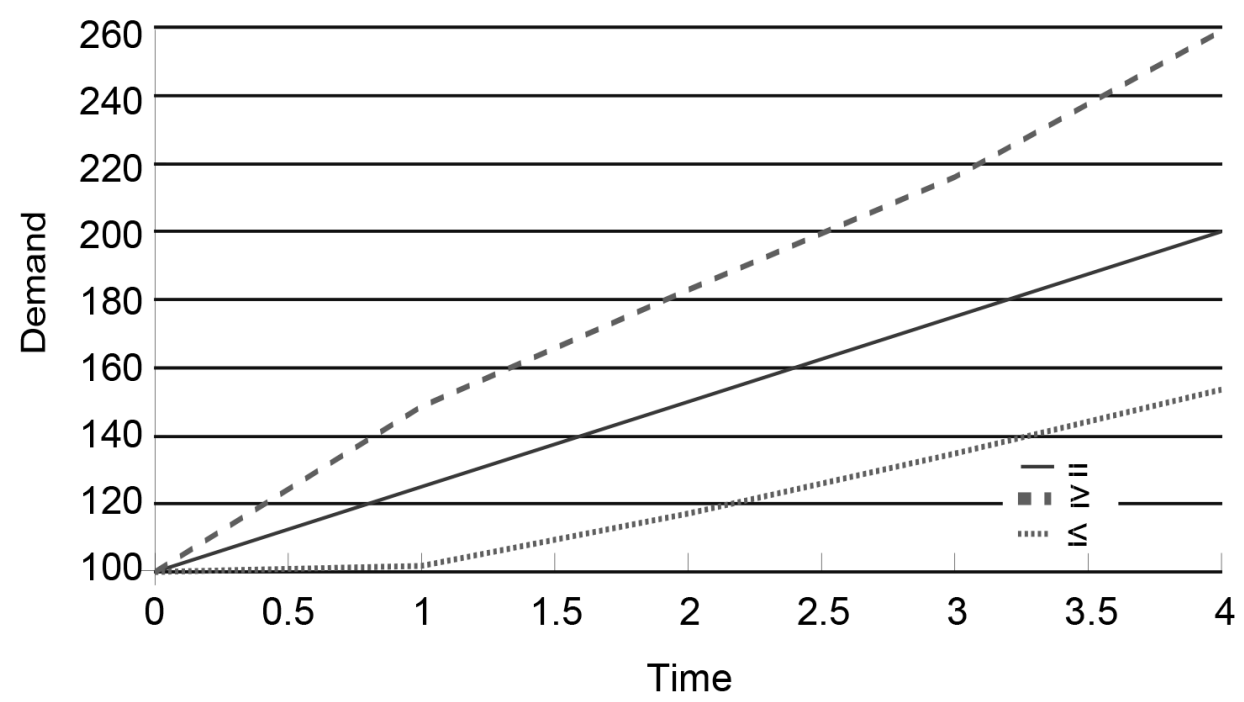

Figure 4 - The demand, according to the three cases of stochastic constraint. 
Figure 5 shows the production rate become higher in the case of stochastic constraint is greater than or equal, due to the increase in the demand rate.

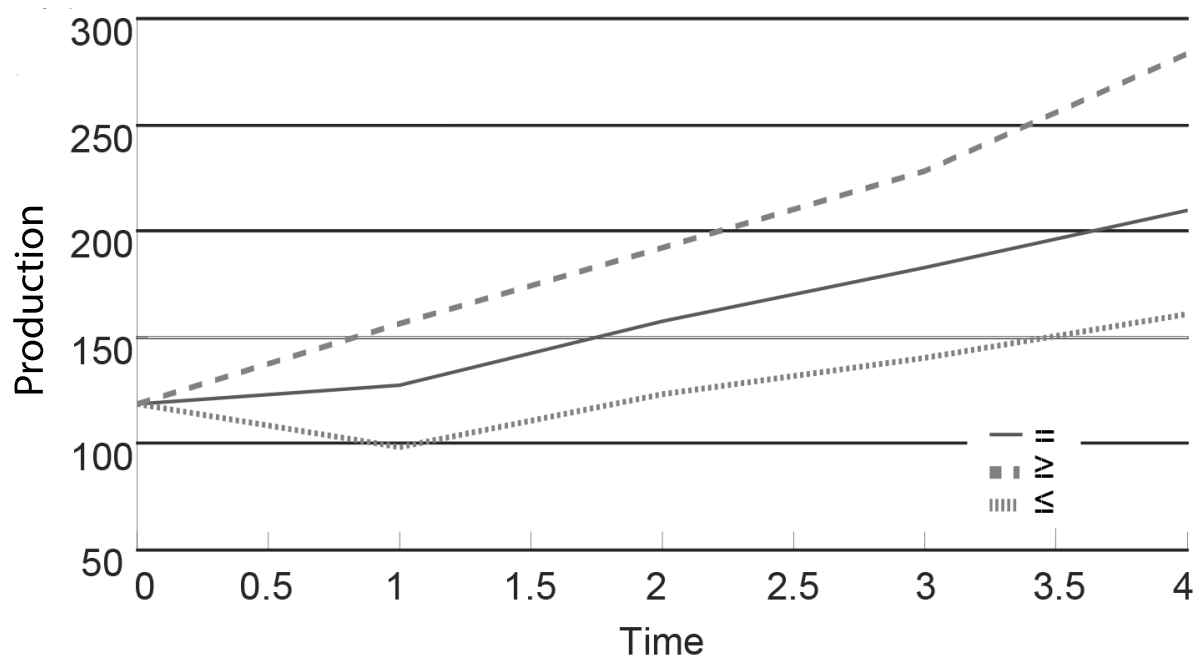

Figure 5 - The production rate, according to the three cases of stochastic demand constraint.

Figure 6 shows the inventory level becoming higher when stochastic constraint is greater than or equal, due to the production rate. The inventory levels at time (1) are similar in all cases, because the inventory, demand, and production at time (0) are similar in all cases (see Figs. 4, 5 and 6).

The total cost of three cases of demand is equal despite a difference in the production rates and inventory levels. This is due to realizing production goal rates and inventory goal levels in all of the cases.

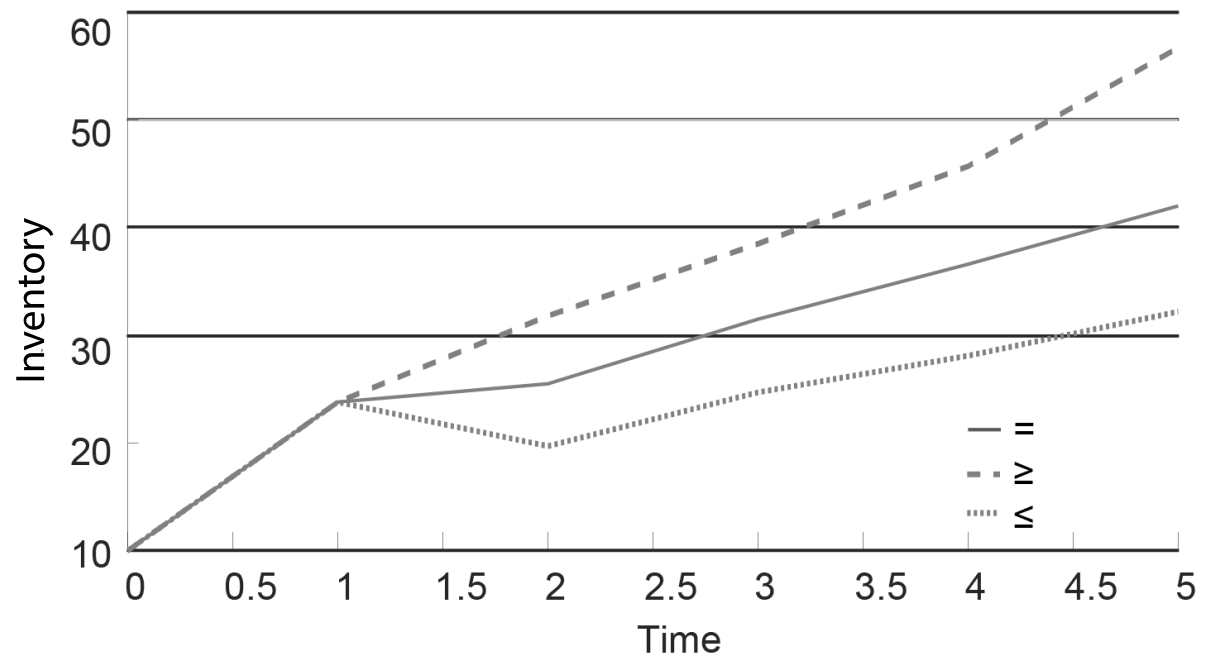

Figure 6 - The inventory level, according to the three cases of stochastic demand constraint. 
Table 3 - Solution of the SQP model with inequality stochastic demand constraint.

\begin{tabular}{|c|c|c|c|c|c|c|c|}
\hline \multirow{2}{*}{ D. } & \multirow{2}{*}{ M.V. } & \multicolumn{7}{|c|}{ Time } \\
\cline { 2 - 8 } & & 0 & 1 & 2 & 3 & 4 & 5 \\
\hline \multirow{3}{*}{$\operatorname{Pr}(D \geq \beta) \geq p$} & $Y$ & 10.00 & 23.75 & 31.40 & 38.33 & 45.27 & 54.76 \\
\cline { 2 - 9 } & $\hat{y}$ & 5.00 & 23.75 & 31.40 & 38.33 & 45.27 & 54.76 \\
\cline { 2 - 9 } & $\hat{n}$ & 118.75 & 156.98 & 191.67 & 226.34 & 273.79 & \\
\cline { 2 - 9 } & $N$ & 118.75 & 156.98 & 191.67 & 226.34 & 273.79 & \\
\cline { 2 - 9 } & $\delta Y$ & 0.00 & 0.71 & 1.88 & 3.45 & 5.43 & 8.21 \\
\hline & $J$ & \multicolumn{7}{|c|}{$125 \$$} & & \\
\hline \multirow{3}{*}{$\operatorname{Pr}(D \leq \beta) \geq p$} & $Y$ & 10.00 & 23.75 & 19.68 & 24.66 & 28.08 & 35.86 \\
\cline { 2 - 9 } & $\hat{y}$ & 5.00 & 23.75 & 19.68 & 24.66 & 28.08 & 32.21 \\
\cline { 2 - 8 } & $\hat{n}$ & 118.75 & 98.40 & 123.31 & 140.38 & 161.03 & \\
\cline { 2 - 8 } & $N$ & 118.75 & 98.40 & 123.31 & 140.38 & 161.03 & \\
\cline { 2 - 8 } & $\delta Y$ & 0.00 & 0.71 & 1.18 & 2.22 & 3.37 & 5.38 \\
\hline & $J$ & \multicolumn{7}{|c|}{$125 \$$} & & \\
\hline
\end{tabular}

\section{CONCLUSION AND RECOMMENDATIONS}

In this paper, two production-inventory system models; optimal control and SQP, were developed. The models took into account stochastic demand, without shortage, and deteriorating items, to achieve the administration goals in the inventory level and hedge demand. Optimal conditions were derived for the optimal control model, along with an explicit solution to the productioninventory model under the periodic-review policy. Furthermore, a comparison between the optimal control model and the SQP model results, in addition to a comparison between all three SQP models, were illustrated.

The SQP model was found to be better than the optimal control model when reaching optimality. At the end of the planning period, the production rate was found to be similar to the other two models. For the SQP model, production rate and inventory level were seen to increase in the case of the stochastic constraint, being greater than or equal. Total cost was controlled for all three SQP models, despite a difference in production rates and inventory levels. These models were found to be economically efficient for inventory control, with stochastic demand and deteriorating items. This study could be extended to include the stochastic holding cost with and without shortage and inventory goal level as a function of demand.

\section{ACKNOWLEDGEMENT}

The author expresses heartfelt gratitude to the referees for their several useful comments and valuable suggestions. 


\section{REFERENCES}

[1] BANeRJee S. 2011. Solution of Stochastic Inventory Models with Chance-Constraints by Intuitionistic Fuzzy Optimization Technique. International Journal of Business and Information Technology, 1(2): $137-150$.

[2] Bhowmick J \& SAmAnta GP. 2012. Optimal inventory policies for imperfect inventory with price dependent stochastic demand and partially backlogged shortages. Yugoslav Journal of Operations Research, 22(2): 199-223.

[3] Castellano D. 2015. Stochastic Reorder Point-Lot Size $(r, Q)$ Inventory Model under Maximum Entropy Principle. Entropy, 18(1): 1-18.

[4] Chotayakul S \& Punyangarm V. 2016. The Chance-Constrained Programming for the LotSizing Problem with Stochastic Demand on Parallel Machines. International Journal of Modeling and Optimization, 6(1): 56-60.

[5] DogRu MK, ReImAn MI \& WANG Q. 2010. A stochastic programming based inventory policy for assemble-to-order systems with application to the W model. Operations research, 58(4-part-1): 849-864.

[6] DostáL Z. 2009. Optimal quadratic programming algorithms: with applications to variational inequalities Vol. 23. Springer Science \& Business Media.

[7] Feng H, Wu Q, Muthuraman K \& Deshpande V. 2015. Replenishment Policies for MultiProduct Stochastic Inventory Systems with Correlated Demand and Joint-Replenishment Costs. Production and Operations Management, 24(4): 647-664.

[8] Germs R \& VAN ForeEst ND. 2013. Optimal control of production-inventory systems with constant and compound poisson demand. University of Groningen, Faculty of Economics and Busisness.

[9] HU M \& YANG Y. 2014. Modified echelon $(r, Q)$ policies with guaranteed performance bounds for stochastic serial inventory systems. Operations Research, 62(4): 812-828.

[10] Hurley G, Jackson P, Levi R, Roundy RO \& Shmoys DB. 2007. New policies for stochastic inventory control models - theoretical and computational results. Working paper, Cornell University, Ithaca, NY.

[11] Karki R, Billinton R \& Verma AK. (Eds.). 2014. Reliability Modeling and Analysis of Smart Power Systems. Springer.

[12] Kazemi Zanjani M, Nourelfath M \& Ait-Kadi D. 2010. A multi-stage stochastic programming approach for production planning with uncertainty in the quality of raw materials and demand. International Journal of Production Research, 48(16): 4701-4723.

[13] KIESMÜLLER GP. 2003. Optimal control of a one product recovery system with lead times. International Journal of Production Economics, 81-82(1): 333-340.

[14] Kim JS \& Jeong WC. 2012. A Coordination Model Under an Order-Up-To Policy. Informatica, 23(2): 225-246.

[15] Kleywegt AJ, Nori VS \& SaVelsbergh MW. 2004. Dynamic programming approximations for a stochastic inventory routing problem. Transportation Science, 38(1): 42-70.

[16] Kumar M, Chauhan A \& Kumar P. 2011. Economic production lot size model with stochastic demand and shortage partial backlogging rate under imperfect quality items. International Journal of Advanced Science and Technology (IJAST), 31(1): 1-22.

[17] KUTZNER SC \& KieSmÜLlER GP. 2013. Optimal control of an inventory-production system with state-dependent random yield. European Journal of Operational Research, 227(3): 444-452. 
[18] Levi R, PÁl M, Roundy RO \& Shmoys DB. 2007. Approximation algorithms for stochastic inventory control models. Mathematics of Operations Research,32(2): 284-302.

[19] Mubiru K. 2014. Optimal Ordering Policies for Inventory Problems in Supermarkets under Stochastic Demand: A Case Study of Milk Powder Product. Proceedings of the 2014 International Conference on Industrial Engineering and Operations Management, Bali, Indonesia: 245-253.

[20] Mubiru K. 2015. Optimal Replenishment Policies for Two-Echelon Inventory Problems with Stationary Price and Stochastic Demand. International Journal of Innovation and Scientific Research, 13(1): 98-106.

[21] OuARet S, Kenné JP \& GHARBi, A. 2011. Hierarchical control of production with stochastic demand in manufacturing systems. AMO - Advanced Modeling and Optimization, 13(3): 419-443.

[22] PAN X \& LI, S. 2015. Optimal control of a stochastic production - inventory system under deteriorating items and environmental constraints. International Journal of Production Research, 53(2): $607-628$.

[23] PréKopA A. 2013. Stochastic programming. Vol. 324. Springer Science \& Business Media.

[24] Raupp F, De Angeli K, Alzamora G \& Maculan N. 2015. Mrp optimization model for a production system with remanufacturing. Pesquisa Operacional, 35(2): 311-328.

[25] REIMAN MI \& WANG Q. 2015. Asymptotically optimal inventory control for assemble-to-order systems with identical lead times. Operations Research, 63(3): 716-732.

[26] Rossi R, TARim SA, Hnich B \& Prestwich S. 2007. Replenishment planning for stochastic inventory systems with shortage cost. In International Conference on Integration of Artificial Intelligence (AI) and Operations Research (OR) Techniques in Constraint Programming. 229-243.

[27] Sethi SP \& Thompson GL. 2000. Optimal Control Theory Application to Management Science and Economics, 2nd ed. USA: Springer.

[28] Shapiro A \& Dentcheva D. 2014. Lectures on stochastic programming: modeling and theory. Vol. 16. SIAM.

[29] SIlva FILho OS. 2014. Optimal Aggregate Production Plans via a Constrained LQG Model. Engineering, 6(12): 773-788.

[30] Soysal MA. 2016. Research on Non-Stationary Stochastic Demand Inventory Systems. International Journal of Business and Social Science, 7(3): 153-158.

[31] TARim SA, Manandhar S \& WAlsh T. 2006. Stochastic constraint programming: A scenariobased approach. Constraints, 11(1): 53-80.

[32] WAnKe P. 2010. The impact of different demand allocation rules on total stock levels. Pesquisa Operacional, 30(1): 33-52.

[33] Wiersema UF. 2008. Brownian motion calculus. John Wiley \& Sons.

[34] Yi F, BAojun B \& Jizhou Z. 2013. The optimal control of production-inventory system. In 25th Chinese Control and Decision Conference (CCDC). China, 4571-4576.

[35] You F \& Grossmann IE. 2011. Stochastic inventory management for tactical process planning under uncertainties: MINLP models and algorithms. AIChE Journal, 57(5): 1250-1277.

[36] Zhang JH, Chen J, Wu YN \& Zhang XS. 2006. A stochastic inventory placement model for a multi-echelon seasonal product supply chain with multiple retailers. In Proceedings of the Sixth International Symposium of Operations Research and Its Applications. Xinjiang, China, 247-257. 